\title{
Hyperhomocysteinemia in recurrent pregnancy loss and the effect of folic acid and vitamin B12 on homocysteine levels: a prospective analysis
}

\author{
Indrani Mukhopadhyay ${ }^{1}$, V. Pruthviraj ${ }^{1}$, Rao P. S. ${ }^{1 *}$, Manash Biswas ${ }^{2}$
}

\begin{abstract}
${ }^{1}$ Department of Obstetrics and Gynecology, Command Hospital AF, Bangalore, Karnataka, India
${ }^{2}$ Department of Obstetrics and Gynecology, Military Hospital, Roorkee, Uttarakhand, India
\end{abstract}

Received: 20 April 2017

Accepted: 24 April 2017

\section{*Correspondence:}

Dr. Rao P. S.,

E-mail: doctorpsrao@gmail.com

Copyright: (c) the author(s), publisher and licensee Medip Academy. This is an open-access article distributed under the terms of the Creative Commons Attribution Non-Commercial License, which permits unrestricted non-commercial use, distribution, and reproduction in any medium, provided the original work is properly cited.

\section{ABSTRACT}

Background: Recurrent pregnancy loss (RPL) affects about 5\% of women. High levels of homocysteine, termed hyperhomocysteinemia, have been implicated in a number of pathologic processes in the venous and arterial vascular systems. Hyperhomocysteinemia in pregnant women has been associated with deep venous thrombosis, recurrent miscarriage, abruption placentae, preeclampsia, neural tube defects, and fetal growth restriction. This study aims at determining association between hyperhomocysteinemia and recurrent pregnancy loss and also association of folic acid (vitamin B 9) and vitamin B 12 with hyperhomocysteinemia (HHCY), in reducing its levels in the body and thus preventing obstetric complications.

Methods: A prospective study of pregnant mothers booked at our hospital over a period of two years with history of unexplained RPL were included in the study and their serum homocysteine levels were assessed. Hyperhomocysteinemia (>12 micromol/1) patients were treated with folic acid and vitamin B12 supplements and homocysteine levels were assessed again, post treatment.

Results: Out of the 100 patients who were assessed, 32\% of RPL patients had hyperhomocysteinemia. Folic acid and VitB12 supplementation reduced homocysteine levels and this was found to be statistically significant.

Conclusions: Hyperhomocysteinemia is associated with RPL. Vitamin supplementation to those with hyperhomocysteinemia, decreases homocysteine levels.

Keywords: Folic acid, Hyperhomocysteinemia, Recurrent pregnancy loss, Vitamin B12

\section{INTRODUCTION}

Spontaneous pregnancy loss can be physically and emotionally taxing for couples, especially when faced with recurrent losses. Recurrent pregnancy loss (RPL), also referred to as recurrent miscarriage or habitual abortion, is historically defined as 3 consecutive pregnancy losses prior to 20 weeks from the last menstrual period (Berry et al, 1995). RPL is also defined by two or more failed consecutive pregnancies. ${ }^{1}$ It is estimated that fewer than $5 \%$ of women will experience two consecutive miscarriages and only $1 \%$ experience three or more. At present, there exists a small number of accepted etiologies for RPL. Most of the diagnosed etiologies include endocrine abnormalities, autoimmune disorders, uterine anomalies, and genetic factors. After evaluation for these causes, approximately half of all cases will still remain unexplained. ${ }^{2}$

Homocysteine is a naturally occurring amino acid that has recently been the subject of much interest. It is involved in several key metabolic processes, including 
the methylation and sulphuration pathways. High levels of homocysteine, termed hyperhomocysteinemia (hhcy), have been implicated in a number of pathologic processes in the venous and arterial vascular systems. The majority of homocysteine is catabolised by the vitamin B6dependent enzyme Cystathione-b-Synthase (CBS) into cystathionine. A significant proportion of homocysteine is regenerated into methionine by methionine synthase. This enzymatic reaction involves methylenetetrahydrofolate reductase (MTHFR) and the cofactor vitamin B12. ${ }^{3}$

Hyperhomocysteinemia can result from a variety of genetic and environmental factors. Genetic factors include importantly the mutations in the MTHFR gene and CBS gene. ${ }^{4}$ The environmental factors being consuming diet poor in folates, vitamin B12. Diets rich in fresh fruits and vegetables as well as vitamin supplements (in particular folic acid) have been associated with lower homocysteine levels. Folates are a group of water soluble vitamins used in the processes of methylation in the body that are implicated in fetal and placental growth. ${ }^{5}$

Hyperhomocysteinemia in pregnant women has been associated with various placental pathologies like RPL, abruption placentae, preeclampsia, fetal growth restriction and stillbirth. ${ }^{6}$ The mechanisms of blood vessel alteration associated with hhcy are still not known perfectly. Homocysteine causes endothelial dysfunction by various mechanisms like nitric oxide inhibition, increasing contractile prostanoids. ${ }^{7}$ The probable mechanism by which hyperhomocysteinemia affects pregnancy and placental implantation is by inhibition of trophoblast functions and cell death.

This study aims to study the role of hyperhomocysteinemia during the early antenatal period as a cause of recurrent pregnancy loss study and also the positive correlation between administration of vitamin supplements with a reduction in homocysteine levels thus reducing the Bad obstetric outcomes with hyperhomocysteinaemia.

Active surveillance of women referred to ANC centres for recurrent pregnancy loss may be helpful to fight this health problem that involves upto $5 \%$ of women of reproductive age by an appropriate treatment. At present as a routine practice, work up in RPL cases does not include test for homocysteine. In case of positive correlation, the test may be recommended as a routine screening test in RPL.

\section{METHODS}

This study was a prospective observational study to know the association between hyperhomocysteinemia and recurrent pregnancy loss and to supplement those mothers with folic acid and vitamin supplements, conducted between Sep 2014 and Aug 2016 at Command Hospital, Air Force, Bengaluru. Total 100 booked cases meeting the inclusion criteria were included in the study. Unexplained two or more consecutive foetal loss in first trimester were included. Patients with pre-existing chronic illness, with multiple pregnancies and presence of an identifiable cause for RPL were excluded. All these patients underwent the test for homocysteine levels at their first visit to the antenatal OPD. They were subsequently monitored closely for evidence of any abnormal pregnancy and maternal outcomes. Plasma tHcy was determined by HPLC (high performance liquid chromatography). Positive results were determined by levels of homocysteine more than $12 \mathrm{micromol} / \mathrm{l}$.

All these pregnant ladies were given tab folic acid $5 \mathrm{mg}$ OD supplementation till termination of pregnancy and inj. Vitamin B12 1mg once a week for 6 weeks. Homocysteine levels were again determined 6 weeks after last dose of inj Vitamin B12 to assess for any decrease in homocysteine levels. Data was compiled using Microsoft Excel and Chi-square test has been used to find the significance of study parameters on categorical scale between two groups.

\section{RESULTS}

In this study of 100 patients with recurrent pregnancy loss whose homocysteine levels were done, $20 \%$ had serum homocysteine values between 1-6 micromol/1, $48 \%$ had serum homocysteine values between 6.1-12 micromol/1, $17 \%$ between 12.1-20 micromol/l and $15 \%$ had homocysteine $>20$ micromol/l. The above values have been depicted in Table 1.

Table 1: Distribution of homocysteine levels in patients studied.

\begin{tabular}{|lll|}
\hline Homocysteine (micromol/l) & $\begin{array}{l}\text { No. of } \\
\text { patients }\end{array}$ & $\%$ \\
\hline $1-6$ & 20 & 20.0 \\
\hline $6.1-12$ & 48 & 48.0 \\
\hline $12.1-20$ & 17 & 17.0 \\
\hline$>20$ & 15 & 15.0 \\
\hline Total & 100 & 100.0 \\
\hline
\end{tabular}

Out of 100 patients, $32 \%$ of pregnant mothers with history of RPL had hyperhomocysteinemia and $68 \%$ of pregnant mothers with history of RPL had normal homocysteine values as has been shown in Table 2 .

Table 2: Incidence of hyperhomocysteinemic patients.

\begin{tabular}{|lll|}
\hline Homocysteine (micromol/l) & No. of patients & $\%$ \\
\hline$\leq 12$ & 32 & 32 \\
\hline$>12$ & 68 & 68 \\
\hline
\end{tabular}

All the antenatal cases who initially had hyperhomocysteinemia, diagnosed in the first visit were given vitamin supplementation. Following this when these patients underwent a repeat investigation for homocysteine levels, $40.6 \%$ had an improvement in 
Homocysteine levels and they reduced to less than 12 micromol/L. However, $59.4 \%$ continued to have hyperhomocysteinemia even after vitamin supplementation. This comparison has been shown in Table 3.

Table 3: Distribution of homocysteine levels after vitamin supplementation.

\begin{tabular}{|lll|}
\hline $\begin{array}{l}\text { Post treatment homocysteine } \\
\text { (micromol/l) }\end{array}$ & $\begin{array}{l}\text { No. of } \\
\text { patients }\end{array}$ & $\%$ \\
\hline$\leq 12$ & 13 & 40.6 \\
\hline$>12$ & 19 & 59.4 \\
\hline Total & 32 & 100 \\
\hline
\end{tabular}

Hence, it was seen that the decrease in homocysteine values after vitamin supplementation that is with Folic Acid and Vitamin B12 in hyperhomocysteinemic patient is statistically significant i.e. $\mathrm{P}$ value $<0.001$. This has been depicted in Table 4.

Hence the following observations were made in present study:

$32 \%$ of pregnant women with history of RPL had hyperhomocysteinemia (about 1 in 3 ).

Folic acid and Vitamin B12 supplementation.

- $40.6 \%$ of those with hyperhomocysteinemia had decrease in homocysteine levels to $<12$ micromol/1 following vitamin supplementation

- The decrease in mean value of homocysteine was by $31.2 \%$ and decrease was statistically strongly significant $(\mathrm{p}$ value $=0.01)$.

Table 4: Comparing pre and post treatment homocysteine levels.

\begin{tabular}{|lllll|l|}
\hline S.Homocysteine $($ micromol/l) & Min-Max & Mean \pm S D & Difference & t value & P value \\
\hline Pre-treatment & $3.2-33.7$ & $11.2 \pm 6.86$ & - & - & - \\
\hline Post-treatment & $3.4-22.6$ & $13.7 \pm 4.2$ & 6.224 & 5.916 & $<0.001$ \\
\hline
\end{tabular}

\section{DISCUSSION}

This study was conducted as a prospective observational study over a period of two years. The main aim of the study was to correlate hyperhomocysteinemia in recurrent pregnancy loss and the effect of folic acid and Vit B12 supplementation on levels of homocysteine.

100 booked antenatal cases with history of recurrent pregnancy loss (two or more consecutive pregnancy loss) and in whom any etiology for RPL (diabetes mellitus, hypothyroidism, and in case of 3 or more recurrent pregnancy loss-APLA screening and parental karyotype) could not be found were considered in the study. Serum homocysteine levels were assessed in the first antenatal visit and those who had hyperhomocysteinemia (>12micromol/l) were given Tab Folic acid $5 \mathrm{mg}$ once daily until delivery and inj. Vit B12 1mg I.M once a week for 6 weeks and homocysteine levels were repeated 6 weeks after last dose of Vit B12, to assess decrease in homocysteine levels post treatment.

Maximum number of all pregnant mothers with history of recurrent pregnancy loss were aged $<30$ years. 87/100 patients were $<30$ and $13 / 100$ patients were $>30$ years of age. $75 \%(75 / 100)$ pregnant ladies had previous two RPL, $22 \%(22 / 100)$ had previous three RPL and 3\% (3/100) had previous four RPL.

In present study $32 \%(32 / 100)$ of all observed patients had hyperhomocysteinemia i.e. about 1 in 3 patients with
RPL had hyperhomocysteinemia, which is in Concordance to the case control study done by Wael Alhalaki and his associates, hhcy was higher among patients with RPL 36/100 (36\%) as compared to control subjects $17 / 100(17 \%)$, the difference was statistically significant $(\mathrm{p}<0.003) .{ }^{4}$ In a study conducted by Maristella et al statistically significant levels of fasting total plasma homocysteine levels were found in those with RPL and unexplained sterility as compared to the control group. The median fasting total plasma homocysteine levels was 19.2 micromol/1 in RPL group as compared to 7.85 micromol/1 in that of study group. ${ }^{8}$ A case-control study by Govindaiah and associates found that maternal hyperhomocysteinemia increases RPL. ${ }^{9}$

In current study $15.6 \% \quad(5$ patients $)$ with hyperhomocysteinemia had first trimester pregnancy loss and $22.1 \%$ (15 patients) with normal homocysteine values had first trimester pregnancy loss. However, this difference was not statistically significant.

$40.6 \%(13 / 32)$ of those who initially had hyperhomocysteinemia, diagnosed in first visit, had normal homocysteine $(<12 \mathrm{micromol} / \mathrm{l})$ values, post vitamin supplementation. $59.4 \%$ of those who initially had hyperhomocysteinemia continued to have hyperhomocysteinemia. (Pre-treatment \pm SD (in hyperhomocysteinemia patients) $=19.92 \pm 5.16$. Post treatment Mean $\pm S D=13.70 \pm 4.27$ ). The decrease in homocysteine levels post vitamin supplementation is 
by $31.22 \%$ and this decrease was found to be strongly significant $(<0.01)$.

In a double blind randomised controlled trial of 12,064 survivors of myocardial ischemia, providing folic acid plus vitamin $\mathrm{B} 12$ reduced homocysteine values by $28 \%(3.8 \mathrm{micromol} / \mathrm{l}){ }^{10}$

In one of the largest trials, a randomised controlled trial done by Homocysteine Lowering Trialists Collaboration in 1998 who assessed the effects of folic acid based supplements on blood homocysteine concentrations on 1114 people, it was found that the proportional and absolute reductions in blood homocysteine produced by folic acid supplements were greater at higher pretreatment blood homocysteine concentrations $(\mathrm{P}<0.001)$ and at lower pre-treatment blood folate concentrations $(\mathrm{P}$ $<0.001)$. After standardisation to pre-treatment blood concentrations of homocysteine of $12 \mathrm{micromol} / \mathrm{l}$ and of folate of $12 \mathrm{nmol} / \mathrm{l}$ (approximate average concentrations for Western populations), dietary folic acid reduced blood homocysteine concentrations by $25 \%$ (95\% confidence interval $23 \%$ to $28 \%$; $\mathrm{P}<0.001$ ), with similar effects in the range of $0.5-5 \mathrm{mg}$ folic acid daily. Vitamin B-12 (mean $0.5 \mathrm{mg}$ daily) produced an additional 7\% (3\% to $10 \%$ ) reduction in blood homocysteine. A meta-analysis in 2005 reaffirmed this. The results of present study were similar to the findings of the above study.

\section{CONCLUSION}

Hyperhomocysteinemia is a risk factor for recurrent pregnancy loss. About 1 in 3 patients of RPL have hyperhomocysteinemia and therefore as a routine workup for RPL serum homocysteine measurement should also be included. Treatment of hyperhomocysteinemia with folic acid and vitamin B12 decreases homocysteine levels significantly.

Funding: No funding sources

Conflict of interest: None declared

Ethical approval: The study was approved by the Institutional Ethics Committee

\section{REFERENCES}

1. Van den Boogaard E, Kaandorp SP, Franssen MT, Mol BW, Leschot NJ, Wouters $\mathrm{CH}$ et al. Consecutive or non-consecutive recurrent miscarriage: is there any difference in carrier status? Hum Reprod. 2010;25:1411-4.
2. Practice Committee of the American Society for Reproductive Medicine. Evaluation and treatment of recurrent pregnancy loss: a committee opinion. Fertil Steril. 2012;98:5.

3. Klai S, Fekih-Mrissa N, El Housaini S, Kaabechi N, Nsiri B, Rachdi R et al. Association of MTHFR A1298C polymorphism (but not of MTHFR C677T) with elevated homocysteine levels and placental vasculopathies. Blood Coagul Fibrinolysis. 2011;22(5):374-8.

4. Alhalaki W, aldin Altanoukhi I, Alhalabi M. The association of hyperhomocysteinemia with recurrent pregnancy loss. Researchgate.net. 2016July;305318605.

5. Mantovani E, Filippini F, Bortolus R, Franchi M. Folic acid supplementation and preterm birth: results from observational studies. Biomed Res Int. 2014;2014:481914.

6. Bergen NE, Jaddoe VW, Timmermans S, Hofman A, Lindemans J, Russcher H, Raat H, SteegersTheunissen RP, Steegers EA. Homocysteine and folate concentrations in early pregnancy and the risk of adverse pregnancy outcomes: the Generation $\mathrm{R}$ Study. BJOG. 2012;119(6):739-51.

7. Cheng ZJ, Yang X, Wang H. Hyperhomocysteinemia and endothelial dysfunction. Curr Hypertens Rev. 2009;5(2):158-65.

8. D'Uva M, Di Micco P, Strina I, Alviggi C, Iannuzzo M, Ranieri A et al. Hyperhomocysteinemia in women with unexplained sterility or recurrent early pregnancy loss from Southern Italy: a preliminary report. Thrombosis J. 2007;5(1):10.

9. Govindaiah V, Naushad SM, Prabhakara K, Krishna PC, Devi AR. Association of parental hyperhomocysteinemia and C677T Methylene tetrahydrofolate reductase (MTHFR) polymorphism with recurrent pregnancy loss. Clini Biochem. 2009;42(4):380-6.

10. Armitage JM, Bowman L, Clarke RJ, Wallendszus $\mathrm{K}$, Bulbulia $\mathrm{R}$, Rahimi $\mathrm{K}$ et al. Effects of homocysteine lowering with folic acid plus Vitamin B12 vs placebo on mortality and major morbidity in myocardial infarction survivors: a randomised trial. JAMA. 2010;303(24):2486-94.

Cite this article as: Mukhopadhyay I, Pruthviraj V, Rao PS, Biswas M. Hyperhomocysteinemia in recurrent pregnancy loss and the effect of folic acid and vitamin B12 on homocysteine levels: a prospective analysis. Int J Reprod Contracept Obstet Gynecol 2017;6:2258-61. 